\title{
EL HUERTO-COMEDOR SUSTENTABLE, UNA EXPERIENCIA DE INTERVENCIÓN EDUCATIVA en la UPN Ayala, Mexico
}

The sustainable school garden-canteen, an experience of educational intervention at UPN Ayala, México

\section{Ricardo Morales Virrueta ${ }^{1}$ \\ Universidad Pedagógica Nacional de México ricardoa.mv@outlook.es}

RESUMEN: Este trabajo nace del proyecto de maestría «El comedor-huerto escolar sustentable. Una experiencia de Intervención Educativa en la UPN Ayala» donde se pone de manifiesto la necesidad de plantear una discusión teórico-práctica en relación con el propio quehacer de la Intervención Educativa. Así mismo se plantea esta propuesta para ponerse en marcha en otros proyectos de la misma naturaleza como son: el cuidado del ambiente, el cuidado de sí mismo, la recuperación de espacios y proyectos estudiantiles. Esta discusión teórico-práctica invita a una reflexión de la forma de hacer Intervención, ya que es importante poner en el centro el contexto donde se espera llevar a cabo el proceso y de esta manera definir si es posible o no hacerlo. Por último, cabe mencionar que esta reflexión fue puesta en marcha con un grupo de trabajo de interventores educativos universitarios y se describen hallazgos y elementos fundamentales para dar cuenta de la utilidad de esta propuesta. Sin embargo, los límites que se encontraron son la dificultad para hacer coincidir la información dada en las páginas gubernamentales, debido a su falta de precisión con los métodos estadísticos que utilizan, la apertura institucional para poner en marcha un proyecto gestionado sólo por estudiantes, así como la visión crítica necesaria de otros interventores para realizar esta contextualización in situ.

Palabras Clave: intervención educativa, ambiente, salud, diagnóstico, dispositivo.

1. Maestro en Educación, Campo: Innovación. 
RESUM: Aquest treball naix del projecte de mestratge «El comedor-huerto escolar sustentable. Una experiencia de Intervención Educativa en la UPN Ayala» on es posa de manifest la necessitat de plantejar una discussió teòric-pràctica en relació amb el propi quefer de la Intervenció Educativa. Així mateix es planteja aquesta proposta per a posar-se en marxa en altres projectes de la mateixa naturalesa com són: la cura de l'ambient, la cura de si mateix, la recuperació d'espais i projectes estudiantils. Aquesta discussió teòric-pràctica convida a una reflexió de la manera de fer Intervenció, ja que és important posar en el centre el context on s'espera dur a terme el procés i d'aquesta manera definir si és possible o no fer-ho. Finalment, cal esmentar que aquesta reflexió va ser posada en marxa amb un grup de treball d'interventors educatius universitaris i es descriuen troballes i elements fonamentals per a donar compte de la utilitat d'aquesta proposta. No obstant això, els límits que es van trobar són la dificultat per a fer coincidir la informació donada en les pàgines governamentals, a causa de la seua falta de precisió amb els mètodes estadístics que utilitzen, l'obertura institucional per a posar en marxa un projecte gestionat només per estudiants, així com la visió crítica necessària d'altres interventors per a realitzar aquesta contextualització in situ.

Paraules clau: intervenció educativa, ambient, salut, diagnòstic, dispositiu.

ABSTRACT: This work is born from the master's project «The sustainable school cafeteria. An experience of Educational Intervention at the UPN Ayala »where the need to raise a theoretical-practical discussion regarding the work of the Educational Intervention is revealed. This proposal is also proposed to be implemented in other projects of the same nature, such as: the care of the environment, the care of oneself, the recovery of spaces and student projects. This theoretical-practical discussion invites a reflection on how doing Intervention, since it is important to put in the centre the context where the process is expected to be carried out and thus define whether or not it is possible to do so. Finally, it is worth mentioning that this reflection was launched with a working group of university educational auditors and fundamental findings and elements are described to account for the usefulness of this proposal. However, the limits that were found are the difficulty to match the information given in the government pages, due to their lack of precision with the statistical methods they use, the institutional opening to launch a project managed 
only by students, as well as the necessary critical vision of other auditors to carry out this contextualization in situ.

KEYWORDS: educational auditor, environment, health, diagnosis, device.

\section{Introducción}

$\mathrm{E}$ ste trabajo nace del proyecto de maestría «El comedor-huerto escolar sustentable. Una experiencia de Intervención Educativa en la UPN Ayala» donde el autor pone de manifiesto la necesidad de plantear una discusión teórico-práctica en relación con el propio quehacer de la Intervención Educativa. Esta discusión se puede lograr haciendo una búsqueda bibliográfica de aquellos autores, científicos sociales, que tuvieron una forma de pensar completamente la vida, la civilización, el trabajo, la libertad, etc. ósea una visión compleja y crítica de la vida humana.

Para empezar este trabajo se describirá, por motivos de espacio, una breve síntesis del planteamiento de entrada: la resignificación o complejización de la noción del dispositivo para la Intervención Educativa, donde uno de los retos más importantes fue la búsqueda de coincidencias entre la postura neoliberal y la visión crítica de teóricos contemporáneos y clásicos, correspondientemente. Luego seguiremos en un intento por aterrizar estos planteamientos y propuestas al grupo de trabajo, donde se explicará cómo se subjetivan, encarnan o se hacen propios los mecanismos de sometimiento capitalista posmoderno, para luego seguir un trabajo de diagnóstico continuo, ya que como se podrá entender un sujeto colectivo o grupo de trabajo recambia y transforma continuamente sus necesidades a través del tiempo, es decir, se trasciende a sí mismo.

En este sentido, en nuestra propuesta se necesita contrastar un dispositivo de referencia, que nosotros entenderemos como el conjunto de prácticas cotidianas, quehaceres, etc. que pertenecen al grupo antes del proceso de intervención, con un dispositivo alternativo que se refiere a la propuesta a transformar en el grupo. Cabe mencionar que esta propuesta, de acuerdo con 
nuestra postura, se construyó colectivamente con el grupo, en voz alta y con acuerdos explícitos de los participantes.

La forma en que se procedió en este trabajo para realizar el diagnóstico fue atendendiendo algunos indicadores:

A. Indicadores socioeconómicos que permitan dar una imagen más amplia y detallada de la comunidad con la que se está trabajando.

B. Elementos de estos indicadores que estén presentes en los quehaceres cotidianos de los integrantes de la comunidad.

C. Las necesidades enunciadas o aquellas que la comunidad identifica en sí misma (autodiagnóstico).

D. Las necesidades que no son dichas, pero se presentan como «problemas» $\mathrm{o}$ «dificultades» para el despliegue del proyecto de acuerdo con los lineamientos o el sentido general del mismo.

Así pues, con los resultados de esta búsqueda y diagnóstico se construyó el dispositivo alternativo, se puso en marcha y continuamente se tuvo una relación dialógica con el grupo de trabajo.

Finalmente se detallarán los hallazgos más importantes para ilustrar la propuesta del presente trabajo dejando al final algunas reflexiones que tienen que ver con la formación de un proyecto de estas características y comentar las necesidades, cualidades y retos de una propuesta alternativa como la nuestra.

\section{Marco teórico-conceptual}

De acuerdo con nuestras investigaciones la noción de «dispositivo» es tratada explícitamente por Agamben (2011) en su conferencia donde a su vez retoma las ideas de Foucault. Esta explicitación junto con el trabajo de Deleuze (1990) coinciden en que los dispositivos están compuestos principalmente por dos elementos, uno de ellos inherente a la naturaleza humana específicamente en su dimensión social y el otro que envuelve estas relaciones dirigiéndolas hacia algún lugar o sentido determinado. Este sentido determinado es para toda relación social un deseo de felicidad que a su vez alimenta y potencia el dispositivo. 
Gracias a estas aproximaciones acerca de la naturaleza de las relaciones sociales, se profundizó haciendo una búsqueda bibliográfica de otros autores que buscaron explicar o hacer propuestas críticas y radicales al respecto, ${ }^{2}$ es decir, obras donde se explican las relaciones de poder entre el desarrollo del hombre y la naturaleza con el fin último de alcanzar la felicidad. Así pues, se encontró que la noción de «trabajo» profundizada en la obra de Marx (1975) tiene implicaciones que ayudan a comprender en principio la esencia de las relaciones sociales y luego en un contexto histórico determinado. Es decir, la noción de trabajo deja, sin lugar a duda, la idea de que el hombre para ser hombre necesita relacionarse con otros hombres, pero también relacionarse de una forma específica con el medio que lo rodea, la naturaleza, y esta forma depende del contexto histórico en que estos hombres, grupos de hombres o civilización en general lo hacen.

Si se hace un recuento histórico todas las formas de organización y desarrollo civilizatorio tienden, tal como la noción del dispositivo, al desarrollo de la felicidad y envuelven las relaciones sociales, tales como el feudalismo, la monarquía, el capitalismo, etc. Luego entonces, la noción del dispositivo ha estado presente en el desarrollo humano se tenga o no consciencia de él.

Ahora bien, en la época moderna y posmoderna, la forma de desarrollo civilizatorio es el capitalismo donde se especifica una manera de realizar trabajo, esto es de forma asalariada y enajeanda al propio trabajador (Marx, 1844) donde, una de sus características principales, es la generación de valor. Si se sigue la obra del capital (Marx, 1975) se dará cuenta que existen dos formas de producir valor bajo el capitalismo y de aumentarlo: a través del detrimento de la calidad de vida de los trabajadores y reduciendo el costo de los medios de producción. Para esta investigación y siguiendo la obra de Foster (1999) y Altvater (2006), la relación que tiene esta forma de producción con la naturaleza da como consecuencias la devastación ambiental, el aumento de enfermedades y la disminución de la calidad de vida de los trabajadores. Esta reflexión pone de manifiesto la dudosa meta del desarrollo civilizatorio como

2. Las obras revisadas donde se tocan estos temas son: El Capital (Marx, 1975), Manuscritos filosóficos y económicos (Marx, 1844), La ética del cuidado de sí como práctica de la libertad (Foucault, 2002), Filosofía de la praxis (Sánchez-Vázquez, 2003), La Intervención Educativa (Remedí, 2004). 
tal, ya que se observa en contraposición con la búsqueda de la felicidad como especie humana, con la posibilidad del desarrollo humano y el inherente ejercicio de su libertar (Foucault, 2002). Cabe destacar que esta forma de trabajo está circunscrita al contexto histórico y no pertenece a la propia naturaleza del trabajo en sí, de manera que es fundamental dar cuenta que no siempre ha sido de esa forma y en consecuencia, no tiene porqué mantenerse así.

Este último punto de enclave fomenta la continua búsqueda de contexto en los trabajos de Intervención - Investigación Educativa (IIE en adelante), por lo que se propone como necesario incluir, en la parte del dispositivo, el contexto específico de la comunidad o grupo con el que se está trabajando para poder analizar qué prácticas o qué dinámicas están interactuando con la lógica capitalista de producción de valor y cuáles tienen otros sentidos. Así mismo, si esta propuesta se construye colectivamente, se puede generar un dispositivo mucho más rico y con apreciaciones más específicas acerca de los modos de vida de los implicados en el proyecto, permitiendo de inmediato el sentido de apropiación de este.

Por otro lado, estas observaciones permiten un punto de vista que se acerca más a explicaciones de raíz para proyectos que tengan relación con el cuidado del medio ambiente, el desarrollo sustentable y la regeneración de la salud, ya que si se transforman las formas de relación hombre-naturaleza, estos problemas pueden resolverse profunda y definitivamente sin que se vean afectados ni el desarrollo social ni la evolución natural.

\section{Diagnóstico grupal}

Todo trabajo de IIE necesita, de entrada, realizar un diagnóstico donde se incluyan los personajes involucrados en el proyecto caracterizándolos. Para este trabajo se buscó apoyo en indicadores sociales y económicos disponibles en línea (e. g. páginas gubernamentales estatales y nacionales) que permiten observar más ampliamente los elementos con los que interactúan los implicados, como una dinámica social. Por otro lado, y para matizar estos datos estadísticos, se realizaron entrevistas estructuradas e informales con la comu- 
nidad estudiantil, los profesores y trabajadores de la institución universitaria, en resumen, se acotó in situ la información gubernamental al sitio de trabajo.

En seguida, se sistematizaron en conjuntos los hallazgos encontrados con la comunidad, siendo primero los indicadores antes mencionados, en segundo lugar, las necesidades vistas desde una perspectiva común, ósea, como proyecto, y por último las necesidades desde una perspectiva de grupo de coordinación a cargo del proyecto en general. Estos niveles o grupos de organización permitieron generar nuevas nociones que enriquecieron el proceso, tales como trabajo cooperativo, apropiación del proyecto, la generación de vínculos de confianza, etc.

El grupo o comunidad de aprendizaje con el que se trabajó estuvo conformado por 15 mujeres jóvenes, de entre 20 y 28 años, universitarias de la Licenciatura en Intervención Educativa en la Universidad Pedagógica Nacional en la Sede de Ayala, en el estado de Morelos México. El grupo se formó a través de invitaciones abiertas y explícitas en la Universidad, carteles y actividades culturales como proyección de videos, talleres, etc. Es importante mencionar que este grupo se conformó también incluyendo al grupo que se encargaba de coordinar el proyecto del Comedor Estudiantil, que llevaba un año de antigüedad.

\section{Indicadores socioeconómicos}

En el estado de Morelos la parte de la población que incluye al conjunto de jóvenes de entre 15 y 28 años, corresponde al 20\%; lo que nos llevó a preguntarnos ¿Porqué es tan baja esta proporción? Esta pregunta nos llevó a los datos de migración (PRONJAG, 2010 y SEDESOL, 2006) que nos ayudaron a explicar que la mayoría de los jóvenes de ese rango de edad han migrado a otros estados en busca de mejores oportunidades laborales y educativas, así como a Estados Unidos para trabajar.

Por otro lado, en el estado sólo el 30\% de la población, en este rango de edad, asiste a alguna institución escolar, de los cuales el 73\% pertenece a educación básica, 16\% media superior y solo el 11\% para educación Universitaria o superior. Ahora bien, en Ayala, el municipio donde se encuentra la Universidad, 49,6\% de la población se encuentra en condiciones de pobreza extrema de acuerdo con datos del CONEVAL (2010). 
Para comprender el contexto de la comunidad con la que se trabajó, se puede decir que la Universidad se encuentra en un municipio donde casi la mitad de la población se encuentra en condiciones de pobreza extrema; este municipio pertenece a uno de los estados con menos oportunidades educativas del país ya que tiene la capacidad sólo de captar al 30\% de la población de jóvenes luego de terminar la educación obligatoria, es decir, la escuela secundaria; orillando así a que estos jóvenes que no pueden tener acceso entren directamente al mercado laboral en el estado, como jornaleros agrícolas, empleados de tiendas comerciales, etc. o migrando a otros lugares con otras ofertas laborales con mejores condiciones.

Estos datos, además de ayudar a describir el contexto de la comunidad con la que se trabajó, serán útiles si se logra encontrar cómo se subjetivan en los individuos, es decir, si se observa cómo se insertan en las prácticas y quehaceres cotidianos de la comunidad, en sus formas y costumbres.

\section{Proyecto}

En esta categoría de análisis podemos decir cuáles eran las necesidades que el grupo quería resolver a través del trabajo en relación con la naturaleza, es decir, aquellas que tienen que ver con la relación entre sujeto-objeto, siendo este último la naturaleza o el medio ambiente con el que se relacionaba.

De esta manera se determinó que la principal necesidad fue la de convertir el comedor en un proyecto sustentable ${ }^{3}$ para establecer una alternativa a la forma de consumo de la comunidad estudiantil. Para esto se decidió que lo mejor era hacer uso de más espacios en la Universidad, habitándolos y dejando para las generaciones futuras estos espacios en manos de los estudiantes. Así, se decidió que el comedor sería abastecido por un huerto que estuviera dentro de la Universidad y fuera trabajado, gestionado y coordinado por los estudiantes; con espacios para estudiar o hacer reuniones y para incluir otros proyectos con sentidos ecológicos y de la salud.

Para lograrlo, se necesitaba hacer una proyección anual que permitiera la distribución de trabajo y actividades en función de la construcción del huerto,

3. Se retomó la noción de desarrollo sustentable «clásica»: El desarrollo que permita resolver las necesidades actuales sin poner en peligro la posibilidad de resolver aquellas de las generaciones futuras. 
herramientas para trabajar en la tierra, contenedores de agua para la época de secas, semillas orgánicas, capacitación para la producción de pesticidas y abonos ecológicos y la delimitación del espacio exclusivo sin que interviniesen otros actores que no fueran estudiantes.

\section{Grupo}

En este caso, se hablará de las necesidades del «sujeto grupal» o la suma de las necesidades individuales de los integrantes del grupo (Traver, 2003) que, a diferencia de la categoría de proyecto, contiene necesidades a nivel subjetivo y resultaron ser en un nivel más emocional que tiene que ver con las relaciones sociales intersubjetivas.

Cabe mencionar que la mayoría de estas necesidades no fueron explicitadas por los integrantes del grupo, sino fueron detectadas por el interventor, el grupo de especialistas asesores y talleristas invitados que interpelaron al grupo de trabajo.

La principal necesidad fue la de incrementar la confianza entre el grupo, pues muchos de los integrantes no se conocían o los que lo hacían habían tenido ya experiencias de trabajo que no necesariamente habían aumentado la confianza, específicamente en la toma de decisiones y la asunción de responsabilidades.

Para intentar comprender y complejizar esta necesidad, diremos que siguió también la dificultad para dialogar abiertamente y con la capacidad de decir las ideas directamente, aunque implicaran diferencias con la coordinación o con todo el demás grupo. En este aspecto, la mayor dificultad era la sensación generalizada de no querer verse mal ante todo el grupo; es decir, ante la distribución de tareas para el proyecto, muchas veces las participantes se comprometían parcialmente frente al grupo, pero en el momento de llevar a cabo la tarea asignada no la hacían o no la entregaban dando razón que realmente no querían o no podían hacerla, pero no habían podido decir «no» frente al grupo.

Y la última de las necesidades era la gestión del espacio y el tiempo con las autoridades del plantel. El grupo trabajaba fuera del horario de clases, pero la Universidad tenía un horario para cerrar sus puertas, generalmente una o dos horas luego de la última clase, y no se permitía que nadie permaneciera 
dentro del plantel. Así mismo algunos maestros, que por cierto no estaban de acuerdo con el proyecto, dejaban muchas actividades extraclase para hacerlas en casa y el tiempo para nuestros encuentros se redujera.

Una vez habiendo diagnosticado estas necesidades, junto con el contexto de los miembros de la comunidad, pasaremos a describir nuestra propuesta como proyecto, ósea, el dispositivo alternativo que fue definido en consenso con el grupo para atender estas necesidades.

\section{Dispositivo alternativo}

Uno de los principales retos para el dispositivo alternativo fue su construcción, pues nuestra postura fue de realizarlo explícitamente con los integrantes del grupo, es decir, contrario a una postura prescrita decidimos que el grupo necesitaba saber qué actividades se iban a realizar, con qué sentido, cómo era la forma de gestionarlas y estar de acuerdo.

Sólo así, nos dimos cuenta que fue necesario darle forma al huerto con medidas, orientación, etc. y fue importante elegir lo que se pretendía cultivar de acuerdo con los cultivos de la región y los que se necesitaban como insumos para el comedor. Estos conocimientos se decidieron trabajar visitando otros huertos estudiantiles en el estado, así como tomar capacitaciones para saber cómo se cultiva la tierra, el cuidado de los cultivos, así como talleres para la preparación de pesticidas y abonos ecológicos.

Por otro lado, también se decidió estudiar y capacitar al grupo para acceder a proyectos que contribuyeran con materiales para trabajar, como palas, azadones, picos, carretillas, contenedores de agua, etc.

En términos del grupo, la necesidad de aumentar la confianza era muy clara así que se decidió que lo mejor para atenderla era teniendo acuerdos claros y respetarlos, que se podía decir "no" a tareas asignadas y que no significaba algo ofensivo en contra del grupo. Así mismo se buscó plantear los principios con los que el grupo tomaba decisiones, actuaba y se regía para que no existiera lugar a dudas acerca del manejo de los recursos, la gestión y responsabilidad con las tareas ni con el equipo de coordinación del proyecto. 
En este sentido también fue importante construir colectivamente la identidad del grupo y a su vez la identidad de los integrantes. Un proyecto de Intervención Educativa realizado con Interventores Universitarios en formación tenía mucho potencial tanto en las metas del proyecto como con actividades que aumentaran la experiencia profesional, dando herramientas del trabajo en campo, que se retroalimentaban en clases, con otros compañeros y finalmente en la vida cotidiana de los participantes.

Finalmente diremos que todo esto era necesario gestionarlo en relación con las autoridades del plantel, pues la mayoría de las actividades se realizarían ahí. Siendo así, los talleres, pláticas, proyecciones de videos, etc. se ordenaron en cronogramas, avisando a las autoridades a través de oficios firmados y sellados por los encargados de recibirlos, y por parte del grupo formar un comité encargado de revisar los avances.

\section{Hallazgos}

Se le nombró hallazgos a lo resultante de la puesta en marcha del dispositivo alternativo con la comunidad, otorgándole un valor importante a aquello que fue enunciado por los participantes de la comunidad en procesos como talleres e interacciones con otros proyectos similares. Estos hallazgos fueron delimitados mediante trabajo colaborativo y comunicación continua entre el grupo de estudiantes y profesores expertos de la Universidad. Estas experiencias se anotaron en libretas de campo del interventor y del grupo de coordinación del proyecto, de forma que al finalizar las sesiones de trabajo se compartían las observaciones entre sí y con el tallerista.

Para empezar, uno de los hallazgos más importantes que se encontró durante la puesta en marcha del dispositivo alternativo fue el que tiene que ver con la coherencia, o falta de ella, de las experiencias que se vivían con las actividades del proyecto y la vida diaria. Si bien es cierto que esto es, a nuestro parecer, una de las dificultades más grandes de cualquier proyecto, y especialmente si tiene carácter educativo, fue muy evidente todo el camino a recorrer para que fuera verdad la intervención. 
Se puede decir que durante las actividades dentro de la Universidad o en las que se llevaron a cabo como visitas a otros huertos y otros comedores en otros lugares, el comportamiento, las decisiones, la postura, las relaciones, etc. estaban en función del cuidado de la salud y del ambiente; se trataba pues de un grupo respetuoso que valoraba aquellos alimentos saludables y los prefería sobre los "chatarra"; apreciaba todas las prácticas de cuidado ambiental, desde la agricultura sustentable hasta el rechazo a envolturas de plástico y alimentos industrializados. Pero estos principios se circunscribían a los momentos de trabajo, ya que una vez concluidas nuestras actividades circulaban los alimentos procesados, refrescos, envolturas de unicel, etc. y en cuanto a las relaciones se apreciaba claramente una pérdida de liderazgo, o más que pérdida un sometimiento ante estudiantes del sexo masculino que dirigían y proponían. En este punto vale la pena recordar que el 100\% de las integrantes del grupo eran mujeres.

Este hallazgo permitió modelar los talleres que se llevaron a cabo, justo para resolver o repensar algunas de estas prácticas grupales. En total se eligieron tres temas de taller: equidad de género, salud y alimentación y huerto.

\begin{tabular}{|l|l|l|l|}
\hline \multicolumn{1}{|c|}{ Taller } & \multicolumn{1}{|c|}{ Duración } & \multicolumn{1}{c|}{ Objetivo } & \multicolumn{1}{c|}{ Contenidos } \\
\hline $\begin{array}{l}\text { Equidad de } \\
\text { género }\end{array}$ & $\begin{array}{l}3 \text { sesiones de } 3 \text { horas c/u, } \\
\text { una por semana }\end{array}$ & $\begin{array}{l}\text { Diferenciar trabajo productivo de } \\
\text { trabajo procreativo entre diferentes } \\
\text { géneros }\end{array}$ & $\begin{array}{l}\text {-Quehaceres domésticos } \\
\text {-Relaciones de poder } \\
\text {-Reflexiones }\end{array}$ \\
\hline $\begin{array}{l}\text { Salud y } \\
\text { alimentación }\end{array}$ & $\begin{array}{l}5 \text { sesiones de 3 horas c/u, } \\
\text { una por semana }\end{array}$ & $\begin{array}{l}\text { Contribuir a la auto reflexión de las } \\
\text { prácticas de alimentación cotidianas }\end{array}$ & $\begin{array}{l}\text {-Alimentos y chatarra } \\
\text {-Contenidos nutricionales } \\
\text {-Creación de menús } \\
\text { saludables } \\
\text {-Alternativas saludables a } \\
\text { platillos cotidianos } \\
\text {-Reflexiones }\end{array}$ \\
\hline Huerto & $\begin{array}{l}5 \text { sesiones de 4 horas } \\
\text { c/u, una por semana } \\
\text { con seguimiento entre } \\
\text { sesiones }\end{array}$ & $\begin{array}{l}\text { Desarrollar a través de la } \\
\text { experiencia las capacidades para } \\
\text { construir un huerto escolar }\end{array}$ & $\begin{array}{l}\text {-Delimitación del espacio } \\
\text {-Diseño estético de plantas } \\
\text {-Interacciones plantas y ser } \\
\text { humano } \\
\text {-Trabajo directo en el huerto }\end{array}$ \\
\hline
\end{tabular}

Tabla 1: Talleres impartidos, duración, objetivos y contenidos desarrollados. Fuente: elaboración propia.

Dentro de los hallazgos encontrados en el primer tema, la equidad de género, estuvieron reflexiones acerca de la salud reproductiva, la forma de tomar decisiones en la escuela y en casa, la forma de asumir las labores domésticas y las prácticas amorosas. Podemos decir que, en conjunto, estas 
reflexiones ayudaron a construir la identidad del grupo y la identidad individual de todos los interventores relacionados con el proyecto, ya que se abrió la posibilidad de darle continuidad a lo vivenciado en las experiencias con nuestra vida diaria.

Sería una contradicción profunda pensar el cuidado de la salud y del ambiente sin tomar en cuenta la complejidad del tema «equidad de género» pues como formas de relacionarlos también se incluyen como determinantes de la salud individual, familiar, grupal y por su puesto social. Lo más destacado del conjunto de estos descubrimientos fue, en principio, encontrar que las mujeres juegan un papel fundamental en la toma de decisiones para el cuidado de la salud reproductiva, ya que la mayoría pensaba que era responsabilidad sólo masculina, y de ahí al poder tomar decisiones como cuándo y con quién tener hijos sólo había un paso.

Es cierto que la mayoría de las integrantes que ya eran madres, no necesariamente habían decidido cuándo tener hijos pues muchas deseaban haberlos tenido después de estudiar su carrera universitaria. Además, justo este tema de ser mujeres universitarias, en su casa, resultaba contradictorio pues se sentían empoderadas, fuertes, con capacidad para liderear proyectos y realizar trabajo cooperativo y comunitario, pero en la Universidad, fuera de ella era difícil ser congruente con esta postura y la postura generalmente machista familiar.

Así mismo el grupo se dio cuenta de la forma de asumir la responsabilidad, lo que como recordaremos generaba desconfianza, no siempre era la mejor. Para ilustrar este punto diremos lo que se vivió en la experiencia: al ser interpeladas ante la pregunta de un tallerista, en casa ¿quién hace las tareas como hacer la comida, limpiar y lavar la ropa? La mayoría respondía que, de los adultos de la casa, pero se dijeron enunciados como «mi marido me ayuda» o «a veces le pido que lo haga porque siempre lo hago yo» que ayudaron a identificar que si alguien «ayuda» ${ }^{4}$ no es su responsabilidad y si no es costumbre el compartir las tareas, no era cierto que fuera tarea de todos. Esto ayudó muchísimo para la forma de asumir responsabilidades en el pro-

4. Fragmento del taller de equidad de género con el especialista Víctor Arizmendi: «¿Qué diferencia hay entre ayudar y hacerse responsable de algo? Por su puesto quien ayuda NO es responsable del trabajo o la tarea. Luego entonces ¿De quién es responsabilidad las tareas domésticas?». 
yecto, pues la mayoría se vivía «ayudando» a los coordinadores del proyecto y, bajo esta lógica, lo más común era olvidar o no poder hacer con lo que se comprometían.

Cabe destacar que el corazón de este descubrimiento fue la diferenciación y su consecuente conceptualización de la forma de trabajo productivo y procreativo: ${ }^{5}$ el trabajo productivo produce valor o ganancia en forma de mercancía o dinero, por ejemplo, el trabajo asalariado; y el trabajo procreativo hace referencia al necesario para realizar la reproducción de los seres humanos, por ejemplo, limpiar la casa, cuidar a los hijos, etc.

Para el segundo punto se hablará a continuación del tema salud y alimentación. Estos temas fueron abordados por otros talleristas especializados en el tema, otras conferencias y discusiones dirigidas por expertos en el tema y chefs.

Con estas experiencias se logró hacer una planeación de los menús para el proyecto del comedor que incluyeron grupos alimenticios que se consideraron saludables, tales como los que pertenecen a las proteínas, vitaminas, carbohidratos y grasas, dando oportunidad a diversificar la oferta. Así mismo se logró reducir los costos de inversión ya que pudo comprar una cantidad mayor de insumos para las comidas de la semana y se dirigió la oferta al consumo de fruta y verdura fresca y cruda como entrada antes de los menús.

Por otro lado, se aprendió a preparar los alimentos más cuidadosamente, a conservarlos mejor y quitar la oferta de bebidas azucaradas, prefiriendo las aguas de sabores naturales y con poca o nada de azúcar añadida.

Finalmente, los menús se organizaron de acuerdo con las necesidades de los estudiantes, en general rapidez, frescura y de fácil digestión, para continuar con energía las clases y de a cuerdo al clima de la región, que es caluroso la mayor parte del año. En conjunto estas prácticas de proyección de menús, cuidado al cocinar y selección de alimentos saludables llegaron a casa de muchos integrantes del grupo, especialmente las madres de familia, que deseaban ofrecer alternativas a sus hijos y esto conllevó a poco a poco

5. Estas nociones fueron trabajadas en el grupo, en forma de discusiones y lectura del texto: Subsunción real del trabajo bajo el capital. 
dejar de consumir alimentos chatarra, industrializados y con poca cantidad de nutrientes.

Para concluir este apartado, se describirán los hallazgos correspondientes al rubro que llamamos huerto.

Los talleres, capacitaciones y visitas para este segmento del dispositivo alternativo se llevaron a cabo con especialistas en el cultivo biointensivo, con especialistas-empíricos con toda su vida de experiencia en el campo que se interesaban en intercambiar conocimientos como la producción orgánica, la producción de pesticidas y abonos ecológicos y la biorremediación y finalmente visitando huertos estudiantiles ya establecidos como el de la Universidad del Estado de Morelos (UAEM), el de la secundaria diurna de Alpuyeca y el huerto de Tenextepango.

Cabe mencionar que parte de las necesidades del huerto era la adquisición de herramientas para trabajar la tierra y contenedores de agua. Esto se resolvió trabajando colectivamente en un proyecto escrito para captar recursos en dos Asociaciones Civiles que se plantearon como objetivo financiar iniciativas sustentables, lo que ayudó mucho a redondear el proceso y también a aterrizar los sueños grupales de construir un huerto a elementos tan básicos como conseguir una pala y aprenderla a usar, aprender a germinar semillas, etc.

Ahora bien, retomando los talleres, capacitaciones y visitas mencionadas, se puede decir que de entrada lo que más llamó la atención del grupo fue la enorme cantidad de trabajo contenida en un proyecto como un huerto escolar. Es decir, en todos los casos se pudo observar que para lograrlo fue necesario incluir por su puesto a los estudiantes, a sus padres de familia, a los maestros, administrativos, trabajadores y actores sociales como fundaciones, asociaciones, etc. pues es una labor muy amplia, que conlleva tiempo, medido en años, y continuidad.

Así mismo, por parte de la gestión con las autoridades del plantel, se logró acordar un espacio determinado y exclusivo para el huerto. Este espacio constaba de unos $180 \mathrm{~m}^{2}$ para uso libre de siembra, construcción de espacios para estudiar y leer, etc. En el tiempo que se llevó a cabo el proyecto, se construyeron dos jardineras de 10X2, un sitio especial para la composta y se sembraron plantas resistentes al calor y desecación, como nopales, árboles y 
algunas plantas medicinales. También se hizo un germinador de semillas resguardado en el comedor escolar para ser usado como instrumento de talleres con niños y otras instituciones.

Por último, se posicionó un depósito de agua de 2000 litros con mangueras y sistema de riego, palas, picos, carretillas y azadones propiedad del proyecto estudiantil, con la consigna de ser usados responsablemente y repuestos en caso de pérdida o rompimiento. También era responsabilidad del equipo de coordinación asegurarse de pasar estas herramientas a otro equipo de generaciones nuevas de universitarios y así sucesivamente.

\section{Reflexiones finales}

Al empezar este trabajo de tesis se explicó la noción de dispositivo, retomando la posición de Agamben (2011) y Foucault (2002) para luego trasponer la noción del trabajo en Marx (1975). De esta manera se propuso incluir la transformación del hombre a través del trabajo, y del contexto a través de la historia mediante diversos dispositivos. Dando como resultado una organización más integral de la información para poder explicar detalladamente las condiciones de referencia en el grupo en un sentido crítico y con claridad. Podemos decir que esto fue importante pues se generó un orden sistemático donde se pueden acomodar datos cuantitativos como índices y censos, así como cualitativos como las entrevistas que buscaron describir la forma de alimentación, las nociones de género y la identidad universitaria en la descripción de la población con la que se trabajó. Es decir, bajo la lógica que se planteó en un inicio de ver al dispositivo en un tiempo y espacio determinados, con una población con características especiales. Así se aterrizaron las ideas de Agamben y Foucault al proyecto, fundamentando nuestras propuestas para explicar e incluir la dinámica del grupo con el que se realizó el proyecto en una dinámica más grande y compleja como la globalización y la subsunción real y formal del consumo bajo el capital.

Esta caracterización in situ permitió definir un dispositivo de referencia específico y adecuado no sólo a la teoría de IIE arriba mencionada a lo largo del texto, sino a las capacidades y necesidades propias del grupo de trabajo. De 
manera se pudo nutrir una parte fundamental del proceso de IIE: el proceso de diagnosis, pues creemos que es una de las partes más compleja y que conlleva más tiempo, ya que representa un reto para cualquier Interventor Educativo, en formación o formado, pues comienza desde el primer acercamiento con la comunidad y termina al cerrar el proyecto, incluyendo por supuesto algunos prejuicios, juicios y etiquetas inexorables, pero tal vez con nuestra propuesta se puedan explicar y contextualizar para intentar afinar el proceso de IIE.

En síntesis, podemos decir que este trabajo, resultado de una experiencia de IIE con la comunidad de estudiantes de la UPN Ayala, busca aportar a la teoría y práctica de la Intervención Educativa una perspectiva más amplia que permita analizar y contextualizar los trabajos de intervención futuros, proponiendo que se incluyan, como parte del dispositivo, el diagnóstico de las características socioeconómicas, índices poblacionales y otros índices ad hoc que el interventor diagnosticará con su trabajo en el campo, así como entrevistas cualitativas que describan más ampliamente a la comunidad con la que se espera trabajar para diseñar colectivamente los términos, expectativas y objetivos del proyecto.

Así que, para finalizarlo, retomaremos el capítulo de la obra de Marx que trata acerca del trabajo. Es necesario recordar que, en esta sección, Marx nos indica algunas nociones muy importantes para reflexionar acerca del trabajo, en sentido amplio, y el trabajo en el sentido de la forma social capitalista. De esta manera diremos que esta forma social es diferente a aquella y ya de entrada nos invita a preguntarnos acerca de la naturaleza del trabajo; o sea, lo que lo hace ser esencialmente trabajo y luego como es que bajo la forma del capitalismo este trabajo es aprovechado para generar más capital. Dentro de la naturaleza del trabajo, nos dice el autor, está una cualidad única y exclusiva de lo humano, que lo separa de cualquier otra actividad animal o vegetal, la consciencia del fin o "la atención" en palabras de Marx. Esta atención es en lo que hemos desde el principio insistido como especificación o afinación del dispositivo, toda vez que se trate de un proyecto de IIE y se busque incluir a la comunidad en la toma de decisiones de este. Cabe mencionar que esto no significa una prescripción de lo que se va a presentar en el trabajo de IIE, o que sea una decisión rígida aplicada al proyecto y no se pueda transformar, sino que a través de la consciencia, el diagnóstico, la experiencia y el acercamiento 
con la comunidad se diseñe, en la esfera de lo ideal, el trabajo terminado antes de comenzar realmente el trabajo de IIE, o sea, imaginar y soñar con algo terminado para poco a poco irnos acercándonos a él si nuestras capacidades nos lo permiten o variarlo y transformarlo cuantas veces sea necesario con las capacidades que tengamos y vayamos desarrollando.

En cuanto a la práctica educativa vale la pena mencionar que la mayoría de las transformaciones que se tuvieron en el grupo pertenecieron al grupo mismo, es decir, a la memoria de la formación del grupo, el sentido y las relaciones que le originaron, así como una de las partes más complejas y que llevó más tiempo: la concientización de las prácticas cotidianas entre los participantes en el grupo. Podemos decir que hacer memoria de la formación del grupo ayudó a que los estudiantes de la Universidad reconocieran el largo trabajo de las compañeras fundadoras y también hacer balance de los alcances que se lograron, así como los límites que se tuvieron. Así mismo ayudó para que otros actores en el proceso, el propio interventor y alumnos de nuevo ingreso pudieran conocer la historia del proyecto en el que se estaban adentrando.

Por otro lado, gracias a este balance se logró redefinir el sentido del grupo, o sea, la intención con la que se mantenían las reuniones semanales para proyectar un cronograma de actividades, talleres y tareas con objetivos y metas bien claras y definidas pues los nuevos participantes lograron aportar y apropiarse del proyecto si se les escuchaba y se incluían sus voces, sueños y deseos.

Dentro de los límites con los que se encuentra esta experiencia, como se mencionó arriba, en muchas ocasiones las instituciones escolares carecen de mecanismos para lograr acoger a los proyectos estudiantiles de esta naturaleza. Se pusieron en juego muchos elementos que corresponden a la lógica de la globalización, como la propiedad de la tierra, la privatización, el trabajo individual, etc., y fue muy complicado recibir ayuda, ya que creemos que no es fácil entender completamente un proyecto de esta magnitud. Tenemos la referencia de otra escuela donde se llevó a cabo un proyecto similar de huerto escolar, solo que a nivel preescolar, la institución intentó asumirlo pero lo hizo de tal manera que recuerda el robo y la privatización; es decir, ya que los alumnos y sus padres de familia se habían organizado para darle vida y 
continuidad al huerto escolar, la institución "tomó posesión" del proyecto, se invirtió en infraestructura para invernadero, se incluyeron pesticidas y abonos inorgánicos así como semillas de dudosa procedencia. Por esto, si bien es cierto que un proyecto de huerto o comedor en manos de los estudiantes puede tener problemas, es necesario que se mantenga así: autónomo e independiente, para que las decisiones, así como la responsabilidad sea de una comunidad estudiantil. Por ello, se propone que los proyectos sustentables necesitan de infraestructura administrativa y económica de la propia escuela, pero a su vez que pueda mantenerse independiente; puede ser una iniciativa de talleres extra clase o incluso en lugar de clases teóricas como medio ambiente y ecología para volverse una práctica de campo continua. Así, de manera natural, la necesidad propia del proyecto encaminará a sus participantes a buscar apoyos académicos y de investigación para avanzar y ésta, a nuestro entender, sería una forma de concretar o realizar la IIE.

\section{Referencias}

Altvater, E. (2006). Existe un marxismo ecológico. Teoría Marxista Hoy, 341-363.

Agamben, G. (2011). ¿Qué es un dispositivo? Sociológica (México), 26(73), 249-264.

Deleuze, G. (1990). ¿Qué es un dispositivo? Michel Foucault, filósofo, 155163.

Foucault, M. (2002). La ética del cuidado de sí como práctica de la libertad en Doce textos fundamentales de la ética del siglo XX. Filosofía Alianza Editorial. México.

Foster, J. B. (1999). Marx's theory of metabolic rift: Classical foundations for environmental sociology. American journal of sociology, 105(2), 366-405.

MARx, K. (1844). El trabajo enajenado en Manuscritos económicos y filosóficos de 1844. Biblioteca Virtual "Espartaco".

Marx, K. (1975). El capital: crítica de la economía política: libro primero. Siglo Veintiuno

REMEDí, E. (2004). La intervención educativa. En Conferencia magistral. Reunión Nacional de Coordinadores de Licenciatura en Intervención Educativa de la Universidad Pedagógica Nacional, México. 
Sánchez Vázquez, A. (2003). Filosofía de la praxis. Editorial Grijalbo. México.

Traver Martí, J. A. (2003). Aprendizaje cooperativo y educación intercultural. Educació intercultural: la diversitat cultural a l'escola, Castelló, UJI Colección educación.

Veraza, J. (2008). Subsunción real del consumo al capital. Editorial Itaca, México. 